10

\title{
Влияние концентрации фосфатов на спектральные свойства ионов тулия во фторфосфатных стеклах
}

\author{
(C) Е.В. Колобкова ${ }^{1,2}$, А. Алхлеф ${ }^{1}$, А.С. Ясюкевич ${ }^{3}$ \\ ${ }^{1}$ Университет ИТМО, \\ 197101 Санкт-Петербург, Россия \\ ${ }^{2}$ Санкт-Петербургский государственный технологический институт (Технический университет), \\ 190013 Санкт-Петербург, Россия \\ ${ }^{3}$ Центр оптических материалов и технологий, Белорусский национальный технический университет, \\ 220013 Минск, Беларусь \\ e-mail: kolobok106@rambler.ru
}

Поступила в редакцию 17.06.2020 г.

В окончательной редакции 15.07.2020 г.

Принята к публикации 28.07.2020 г.

\begin{abstract}
Рассчитаны параметры Джадда-Офельта для ионов тулия во фторофосфатных стеклах при изменении содержания фосфатной компоненты матрицы. Показана близость значений параметров $\Omega_{t}$ стекол с содержанием до $10 \mathrm{~mol} \% \mathrm{Ba}\left(\mathrm{PO}_{3}\right)_{2}$ к аналогичным параметрам фторидных стекол и резкое увеличение их значений при увеличения фосфатов. В рамках теории Джадда-Офельта проведен анализ спектральнолюминесцентных параметров стекол. Получены вероятности спонтанного перехода, коэффициенты ветвления $(\beta)$ и радиационные времена жизни $\left(\tau_{\text {rad }}\right)$ для различных возбужденных состояний $\mathrm{Tm}^{3+}$. Обнаружено, что при малых концентрациях фосфатов для стекол характерно высокое радиационное время жизни возбужденного лазерного уровня ${ }^{3} F_{4}$. Показано, что для исследованных стекол характерен широкий плоский спектр коэффициента усиления, что делает их перспективными материалами для создания широкополосных усилителей.
\end{abstract}

Ключевые слова: ионы $\mathrm{Tm}^{3+}$, параметры Джадда-Офельта, поглощение, усиление, сечение вынужденного излучения.

DOI: $10.21883 /$ OS.2020.12.50329.176-20

\section{Введение}

Интерес к спектральному диапазону $1.8-2 \mu \mathrm{m}$ связан с необходимостью создания мощных источников света для лазерных локаторов ИК диапазона (ЛИДАР), лазеров для медицинских и военных приложений [1-3]. В качестве активных сред для работы в этом диапазоне могут быть использованы кристаллы и стекла, активированные ионами гольмия или тулия. В связи с этим появилось большое число работ, посвященных стеклам различных составов, активированных ионами $\mathrm{Tm}^{3+}$, для создания волоконных лазеров, работающих на длине волны $2000 \mathrm{~nm} \mathrm{[4-8].} \mathrm{Большим} \mathrm{преимушеством} \mathrm{иона}$ тулия является возможность накачки диодным лазером с $\lambda=800 \mathrm{~nm}[4]$.

При выборе матрицы стекла для активации ионами тулия с целью получения на их основе активных элементов необходимо учитывать спектральный диапазон пропускания, характерный для разного вида стекол. С точки зрения этой характеристики для активации ионами тулия перспективны прежде всего теллуритные, германатные и фторидные стекла, чье пропускание сдвинуто в длинноволновую область спектра [5-7]. Однако у каждой из перечисленных матриц есть определенные недостатки. Теллуритные стекла характеризуются низкими физикохимических параметрами, а германатные стекла имеют высокую стоимость. Традиционными лазерными матрицами являются силикатные и фосфатные стекла $[4,5,8]$, которые ранее хорошо зарекомендовали себя как лазерные среды для других активаторов. Однако в оксидных стеклах всегда присутствует определенная концентрация $\mathrm{OH}$-групп, которые являются основной тушащей примесью редкоземельных ионов. Помимо присутствия ОНгрупп, сетка традиционных лазерных фосфатных и силикатных стекол характеризуется высокими энергиями фононов, что увеличивает вероятность многофононной релаксации и уменьшает квантовый выход флуоресценции. Фторидные стекла лишены этих недостатков. Для них характерны низкие энергии фононов, что приводит к расширению пропускания в ИК области до 7.0 $\mu \mathrm{m}[9]$. Однако фторидные стекла различных составов имеют высокую склонность к кристаллизации.

Известно, что введение во фтороалюминатное стекло небольших добавок фосфатов стабилизирует сетку стекла и позволяет получать стекла оптического качества в широком диапазоне концентраций активатоpa [10]. Ранее нами были рассмотрены особенности спектрально-люминесцентных характеристик фторофосфатных стекол, активированных ионами неодима и парой $\mathrm{Er}^{3+} / \mathrm{Yb}^{3+}$ [11-14]. Оказалось, что фторофосфатные стекла с малыми добавками фосфатов имеют спектральнолюминесцентные параметры, близкие к параметрам 
фторидных стекол, и физико-химические свойства, которые сопоставимы со свойствами оксидных стекол. Анализ влияния увеличения фосфатной компоненты на спектрально-люминесцентные характеристики ионов неодима и эрбия показал слабое влияние фосфатов в диапазоне $2-10 \mathrm{~mol} . \% \mathrm{Ba}\left(\mathrm{PO}_{3}\right)_{2}$ и радикальное изменение спектрально-люминесцентных параметров при повышении их содержания. Преимущества фторидной и фторофосфатной матриц, активированных редкоземельными ионами для создания активных сред, были детально рассмотрены в $[15,16]$.

В предлагаемой работе на основании теории Джадда-Офельта проведен анализ спектрально-люминесцентных характеристик фторофосфатных стекол, активированных ионами тулия в зависимости от концентрации фосфатной компоненты.

\section{Объект исследования и методика эксперимента}

Были синтезированы стекла с переменным содержанием фосфатной компоненты в системе $x \mathrm{Ba}\left(\mathrm{PO}_{3}\right)_{2}-$ $-(99-x)\left(\mathrm{AlF}_{3}-\mathrm{CaF}_{2}-\mathrm{MgF}_{2}-\mathrm{BaF}_{2}-\mathrm{SrF}_{2}\right)-1 \mathrm{TmF}_{3}$ (где $x=2 ; 5 ; 10 ; 20 ; 30$ mol.\%). Выбранная концентрация $\mathrm{TmF}_{3}$ является оптимальной для анализа влияния состава матрицы на люминесцентные свойства. При этой концентрации во фторофосфатных стеклах уже наблюдаются ион-ионные взаимодействия, в частности кроссрелаксация, которая является эффективным механизмом увеличения заселенности верхнего лазерного уровня $[17,18]$.

Синтез проводился в закрытых стеклоуглеродных тиглях в атмосфере аргона при температуре $1050^{\circ} \mathrm{C}$ в течение $30 \mathrm{~min}$. Навеска составляла $50 \mathrm{~g}$. Гомогенный расплав вырабатывался на гладкую стеклоуглеродную пластину и отжигался при температуре $440^{\circ} \mathrm{C}$ вблизи температуры стеклования. Температуры стеклования $\left(T_{g}\right)$ были определены на основании анализа кривых ДСК, полученных на дифференциальном сканирующем калориметре (STA 449F1 Jupiter Netzsch), сканирование проводилось со скоростью $10 \mathrm{~K} / \mathrm{min} . T_{g}$ составляли $436-450^{\circ} \mathrm{C}$ в зависимости от состава стекла. Спектры

Таблица 1. Физические свойства фторофосфатных стекол, активированных ионами тулия с различным содержанием фосфатов

\begin{tabular}{l|c|c|c|c|c}
\hline Образец & $\begin{array}{c}\mathrm{TmF}_{3}, \\
\mathrm{~mol} \%\end{array}$ & $\begin{array}{c}\mathrm{Ba}\left(\mathrm{PO}_{3}\right)_{2}, \\
\mathrm{~mol} \%\end{array}$ & $\begin{array}{c}\rho, \\
\mathrm{g} / \mathrm{cm}^{3}\end{array}$ & $\begin{array}{c}N_{\mathrm{Tm}}, \\
10^{20} \mathrm{~cm}^{-3}\end{array}$ & $n_{D}$ \\
\hline$P 2$ & 1 & 2 & 3.935 & 2.29 & 1.434 \\
$P 5$ & 1 & 5 & 3.937 & 2.16 & 1.450 \\
$P 10$ & 1 & 10 & 3.939 & 1.99 & 1.463 \\
$P 20$ & 1 & 20 & 3.943 & 1.71 & 1.504 \\
$P 30$ & 1 & 30 & 3.955 & 1.50 & 1.500
\end{tabular}
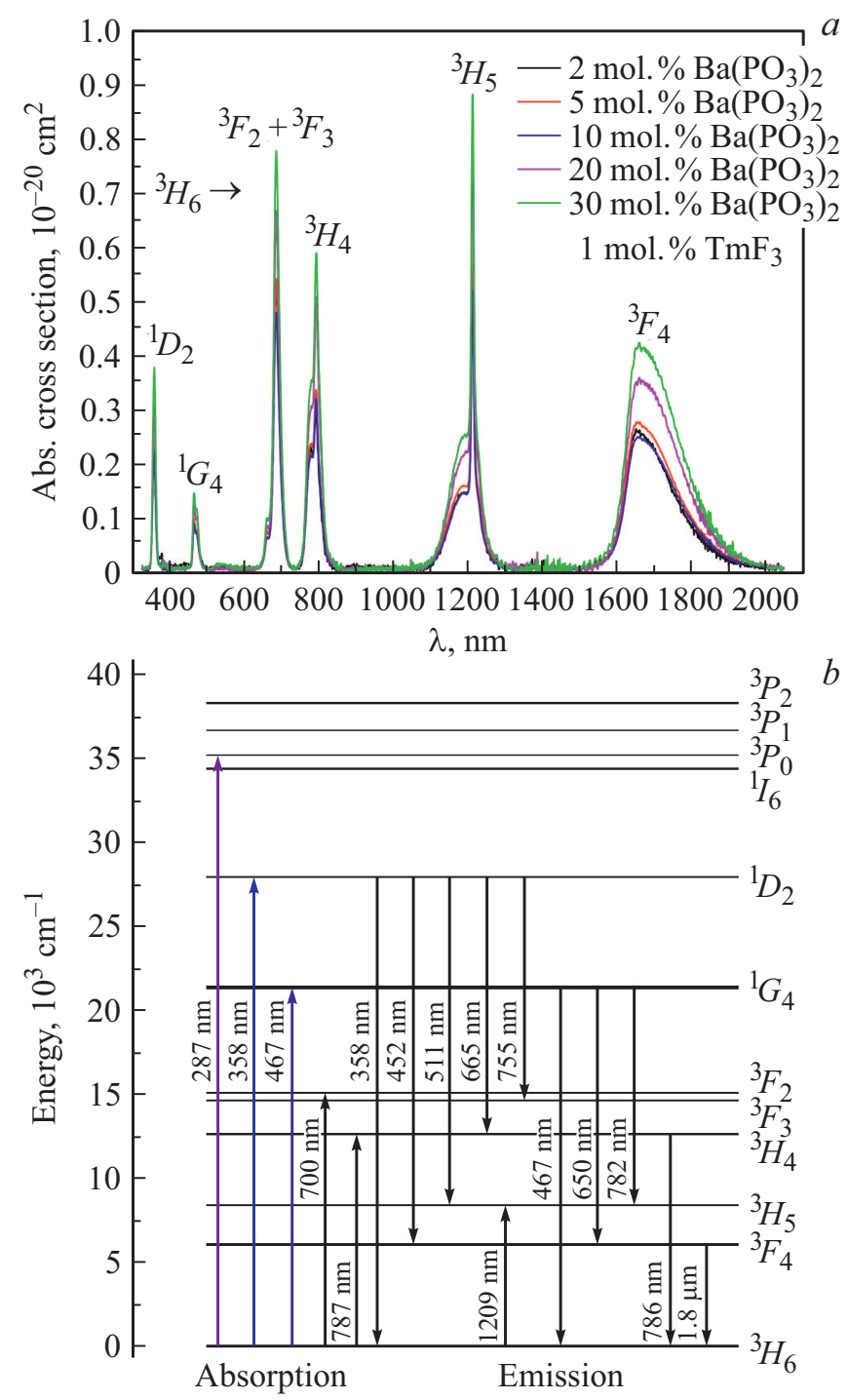

Pис. 1. (a) Сечение поглощения для стекол, активированных ионами $\mathrm{Tm}^{3+}$, при различных концентрациях фосфата. (b) Диаграмма энергетических состояний и основных переходов иона тулия.

поглощения полированных плоскопараллельных образцов регистрировались с помощью спектрофотометров Lambda 650 и Lambda 900 (PerkinElmer) в диапазонах длин волн 300-900 nm и 900-2050 nm соответственно. Плотность стекол определили гидростатическим взвешиванием с точностью $10^{-3} \mathrm{~g} / \mathrm{cm}^{3}$. Показатель преломления измеряли с помощью рефрактометра Аббе IRF454 BM, работающего на линиях спектра $F, D, C$.

\section{Экспериментальные результаты и обсуждение}

Спектры поглощения и параметры ДжаддаОфельта. Молярная концентрация $\mathrm{Ba}\left(\mathrm{PO}_{3}\right)_{2}$, плотность $(\rho)$, показатель преломления $(n)$ и концентрация 
ионов $\mathrm{Tm}^{3+}\left(N_{\mathrm{Tm}}\right)$ исследуемых стекол представлены в (табл. 1).

Спектры сечений поглощения стекол, активированных ионами $\mathrm{Tm}^{3+}$, представлены на рис. $1, a$. Анализ сечений поглощения показал их практическую неизменность в диапазоне 2-10 mol.\% $\mathrm{Ba}\left(\mathrm{PO}_{3}\right)_{2}$ и заметный рост при дальнейшем увеличении содержания фосфатов. Диаграмма энергетических уровней иона тулия показана на рис. $1, b$. Все наблюдаемые переходы и соответствующие им полосы поглощения являются переходами из основного состояния ${ }^{3} \mathrm{H}_{6}$ на возбужденные уровни.

Параметры Джадда-Офельта (Д-О) рассчитываются путем минимизирования среднеквадратического отклонения (СКО) между измеренными и расчетными силами линий дипольного электрического перехода. Расчетная сила линии рассчитывается по формуле:

$$
S_{\text {ed }}^{\text {calc }}\left(J \rightarrow J^{\prime}\right)=\sum_{t=2,4,6} \Omega_{t}\left|\left\langle\Psi_{J}\left\|U^{t}\right\| \Psi_{J^{\prime}}^{\prime}\right\rangle\right|^{2},
$$

где коэффициенты $\Omega_{t} \quad-\quad$ это параметры Джадда-Офельта, $\left|U_{t}\right|^{2}-$ квадраты приведенных матричных элементов, рассчитанные в [19].

Измеренная сила линии рассчитывается по формуле

$$
S_{\mathrm{ed}}^{\text {meas }}\left(J \rightarrow J^{\prime}\right)=\frac{27 h c(2 J+1) n}{8 \pi^{3} \bar{\lambda} e^{2}\left(n^{2}+2\right)^{2}} \int \sigma_{a b s}(\lambda) d \lambda,
$$

где $h$ - постоянная Планка, $c$ - скорость света в вакууму, $e-$ заряд электрона, $n-$ показатель преломления, $\bar{\lambda}-$ средняя длина волны полосы $(\mathrm{nm}), J$ и $J^{\prime}$ - значение полного момента начального и конечного состояний.

Точность подгонки Д-О параметров может быть выражена через СКО $(\Delta r m s)$ с помощью уравнения (3)

$$
\Delta r m s=\left[\frac{\sum\left(S_{\mathrm{ed}}^{\mathrm{meas}}-S_{\mathrm{ed}}^{\mathrm{calc}}\right)^{2}}{p-q}\right]^{\frac{1}{2}},
$$

где $p$ - число полос поглощения, входящих в расчеты Д-О, а $q$ - число параметров интенсивности. В этой работе $p=6, q=3$.

Погрешность подгонки выражается в виде отношения СКО к среднему значению по всем используемым измеренным силам линий. Погрешность расчета параметров указывается в процентах [20].

Измеренные силы линий и параметры интенсивности $\Omega_{t}(t=2,4,6)$ для стекол с переменным содержанием фосфатов приведены в табл. 2, расчетные силы линий, СКО и погрешность подгонки приведены в табл. 3. На рис. 2 показана зависимость параметров Д-О при изменении содержания фосфатов в стекле.

Изменения значений параметров Д-О имеют жесткую корреляцию с вариацией ближайшего окружения P3 ионов $[21,22]$. На величину $\Omega_{2}$ влияет симметрия поля лигандов и степень ионности/ковалентности связи активатор-лиганд. Ионы кислорода обладают более высокой поляризуемостью, чем ионы фтора. Известно,
Таблица 2. Измеренная сила линии $\left(S_{\mathrm{ed}}^{\text {calc }}\right)\left(10^{-20} \mathrm{~cm}^{2}\right)$ для переходов из основного состояния ${ }^{3} H_{6}$ в конечные состояния и параметры Д-О

\begin{tabular}{l|c|c|c|c|c}
\hline $\mathrm{Ba}\left(\mathrm{PO}_{3}\right)_{2}$, mol. $\%$ & 2 & 5 & 10 & 20 & 30 \\
\hline${ }^{3} F_{4}$ & 2.4434 & 2.7115 & 2.4339 & 3.5613 & 4.1716 \\
\hline${ }^{3} H_{4}$ & 1.2888 & 1.4203 & 1.3224 & 1.9961 & 2.2739 \\
\hline${ }^{3} F_{2}+{ }^{3} F_{3}$ & 1.8041 & 1.8108 & 1.5681 & 2.1319 & 2.4698 \\
\hline${ }^{1} G_{4}$ & 0.3213 & 0.3035 & 0.2823 & 0.3903 & 0.4481 \\
\hline${ }^{1} D_{2}$ & 0.7572 & 0.7127 & 0.6313 & 0.8671 & 1.0051 \\
\hline$\Omega_{2}\left(10^{-20} \mathrm{~cm}^{2}\right)$ & 1.55 & 2.14 & 2.10 & 3.48 & 4.00 \\
\hline$\Omega_{4}\left(10^{-20} \mathrm{~cm}^{2}\right)$ & 1.89 & 1.80 & 1.50 & 1.93 & 2.32 \\
\hline$\Omega_{6}\left(10^{-20} \mathrm{~cm}^{2}\right)$ & 1.12 & 1.15 & 1.02 & 1.44 & 1.63 \\
\hline$\Omega_{4} / \Omega_{6}$ & 1.69 & 1.57 & 1.47 & 1.34 & 1.42
\end{tabular}

Таблица 3. Расчетная сила линии $S_{\text {ed }}^{\text {calc }}\left(10^{-20} \mathrm{~cm}^{2}\right)$, $\mathrm{CKO}\left(10^{-20} \mathrm{~cm}^{2}\right)$ и погрешность расчета

\begin{tabular}{l|c|c|c|c|c}
\hline $\mathrm{Ba}\left(\mathrm{PO}_{3}\right)_{2}$, mol. $\%$ & 2 & 5 & 10 & 20 & 30 \\
\hline${ }^{3} F_{4}$ & 2.473 & 2.731 & 2.462 & 3.6131 & 4.2222 \\
\hline${ }^{3} H_{4}$ & 1.239 & 1.386 & 1.269 & 1.8909 & 2.1716 \\
\hline${ }^{3} F_{2}+{ }^{3} F_{3}$ & 1.8299 & 1.828 & 1.596 & 2.1889 & 2.5252 \\
\hline${ }^{1} G_{4}$ & 0.2303 & 0.252 & 0.226 & 0.3303 & 0.3870 \\
\hline${ }^{1} D_{2}$ & 0.7012 & 0.673 & 0.568 & 0.7413 & 0.8829 \\
\hline СКО & 0.088 & 0.055 & 0.076 & 0.136 & 0.13 \\
\hline Погрешность, \% & 6.6 & 3.9 & 6.0 & 7.5 & 6.3
\end{tabular}

что при замещении ионов фтора на ионы кислорода ковалентность связи между $\mathrm{Tm}^{3+}$ и анионами из первой координационной сферы увеличивается, а $\Omega_{2}$ возрастает [21]. Вторым фактором, влияющим на величину $\Omega_{2}$, является симметрия поля лигандов, уменьшение которой также приводит к увеличению $\Omega_{2}$. Однако фосфатные группы играют особую роль в изменении доли ковалентности связи Р3 ионов с лигандами. Было обнаружено, что увеличение содержания фосфатов имеет два противоположных воздействия на $\Omega_{2,6}$ в отличие от других оксидных систем. Появление фосфатных групп в стекле приводит к увеличению электронной плотности от $2 p$-орбиталей ионов кислорода, а также донорству $\pi$-электронов от $\mathrm{P}-\mathrm{O} p$-связи. Первый эффект приводит к увеличению $\Omega_{2}$, но уменьшению $\Omega_{6}$. Напротив, большее донорство $\pi$-электронов от фосфатных групп увеличивает $\Omega_{6}$.

Сопоставление этих закономерностей с нашими результатами, представленными на рис. 3 , показывает, что 
Таблица 4. Параметры $\Omega_{t}\left(10^{-20} \mathrm{~cm}^{2}\right)$ для $\mathrm{Tm}^{3+}$ в различных стеклах

\begin{tabular}{l|c|c|c}
\hline Стекло & $\Omega_{2}$ & $\Omega_{4}$ & $\Omega_{6}$ \\
\hline Силикатное [4] & 3.08 & 0.99 & 0.40 \\
Фторидное (ZBLAN) [5] & 1.92 & 1.68 & 1.13 \\
Фторофосфатное [5] & 2.75 & 2.28 & 1.18 \\
Теллуритное [6] & 4.48 & 1.87 & 1.03 \\
Германатное [7] & 7.32 & 1.88 & 2.86 \\
Фосфатное [8] & 5.63 & 1.75 & 1.11 \\
Фторофосфатное P5 (данная работа) & 2.14 & 1.80 & 1.15 \\
Фторофосфатное P30 (данная работа) & 4.00 & 2.32 & 1.63
\end{tabular}

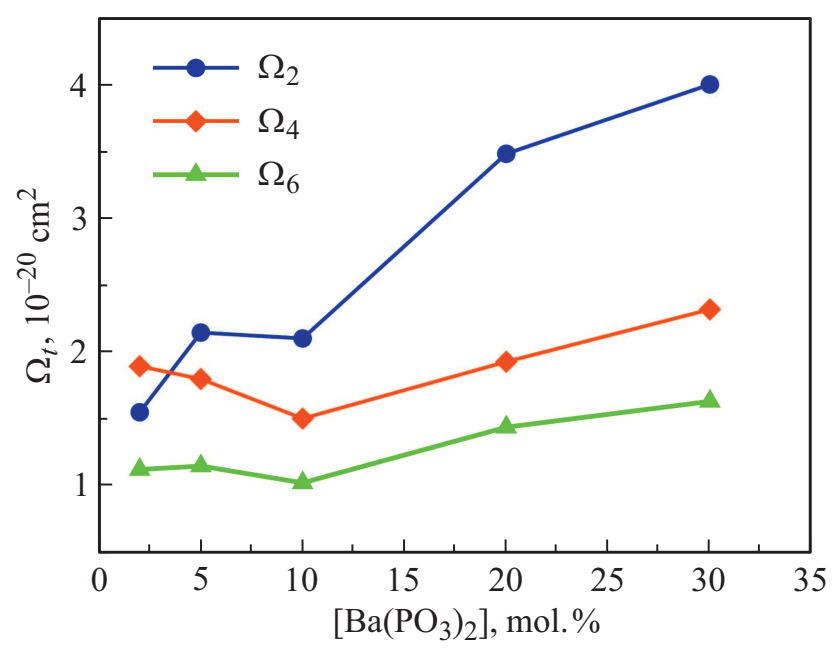

Рис. 2. Зависимость параметров Д-О от содержания фосфатов в стекле.

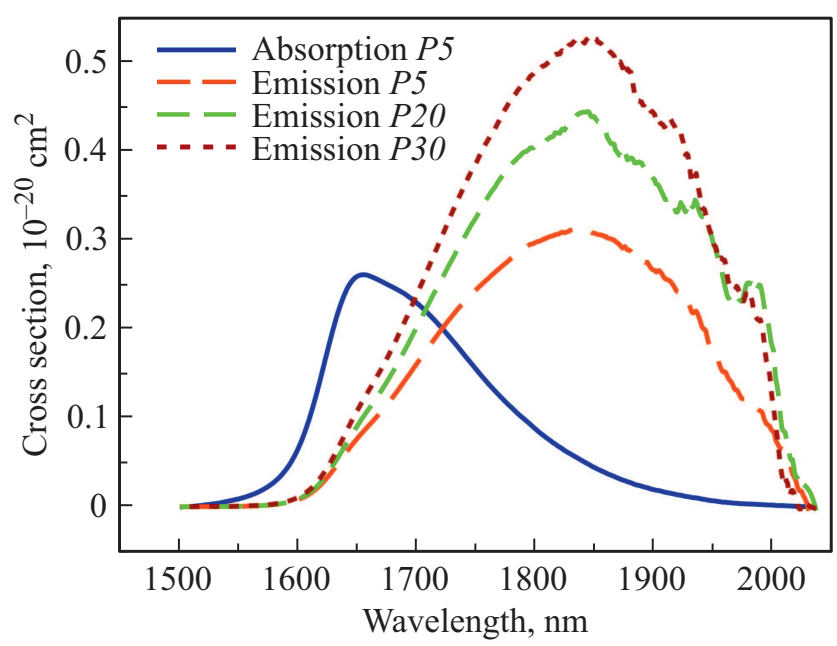

Рис. 3. Сечения поглощения стекла $P 5(a)$ и вынужденного излучения стекол $P 5, P 20, P 30$.

второй эффект превалирует и происходит увеличение параметра $\Omega_{6}$ при увеличении содержания $\mathrm{Ba}\left(\mathrm{PO}_{3}\right)_{2}$. В табл. 4 параметры Д-О для фторофосфатных стекол с малым $(P 5)$ и большим $(P 30)$ содержанием фосфатов сопоставлены с параметрами других стекол, легированных ионами $\mathrm{Tm}^{3+}$. Видно, что при малом содержании фосфата параметры $\Omega_{2}$ и $\Omega_{6}$ для изучаемых стекол близки к параметрам фторидных стекол, легированных $\mathrm{Tm}^{3+}$, и заметно изменяются при увеличении содержания фосфатной составляющей.

Люминесцентные свойства. Вероятность радиационного перехода между двумя состояниями (с $J$ на $J^{\prime}$ ), $A\left(J-J^{\prime}\right)$, можно получить, используя расчетную силу линии, из соотношения (4)

$$
A\left(J \rightarrow J^{\prime}\right)=\frac{64 \pi^{4} e^{2}}{3 h(2 J+1) \bar{\lambda}^{3}}\left[n\left(\frac{n^{2}+2}{3}\right)^{2} S_{\text {ed }}^{\text {calc }}\right] .
$$

Радиационное время жизни $\left(\tau_{\text {rad }}\right)$ для верхнего метастабильного состояния можно рассчитать по формуле

$$
\tau_{\mathrm{rad}}=\frac{1}{\sum A\left(J \rightarrow J^{\prime}\right)} .
$$

С другой стороны, коэффициенты ветвления флуоресценции $(\beta)$ выражаются как отношения вероятностей радиационных переходов к сумме вероятностей всех радиационных переходов следующим образом:

$$
\beta\left(J \rightarrow J^{\prime}\right)=\frac{A\left(J \rightarrow J^{\prime}\right)}{\sum A\left(J \rightarrow J^{\prime}\right)} .
$$

В табл. 5 приведены расчетные прогнозируемые вероятность спонтанного перехода $(A)$, общая вероятность спонтанного перехода $\left(\sum A\right)$, радиационное время жизни $\left(\tau_{\text {rad }}\right)$ и коэффициенты ветвления $(\beta)$ оптических переходов для образца стекла $P 5$, легированного $\mathrm{Tm}^{3+}$.

Расчёты показали (табл. 5), что радиационное время жизни для перехода ${ }^{3} F_{4} \rightarrow{ }^{3} H_{6}$ иона $\mathrm{Tm}^{3+}$ в стекле $P 5$ составляет $8.54 \mathrm{~ms}$, что значительно превышает аналогичный параметр, рассчитанный для ранее исследованных фторофосфатных стекол: 4.86 и $4.44 \mathrm{~ms}$ соответственно $[23,24]$. Следует отметить, что увеличение времени жизни верхнего лазерного уровня позволяет уменьшить порог генерации [24].

Сравнение параметров люминесценции для стекол с переменным содержанием фосфатов представлено в табл. 6. Показано, что при увеличении содержания фосфатов время жизни метастабильного состояния ${ }^{3} F_{4}$, уменьшается примерно от 10 до $5 \mathrm{~ms}$.

Сечение вынужденного излучения было рассчитано при использовании модифицированного метода соответствия для аморфных изотропных сред (МMC) [25] по формуле

$$
\begin{aligned}
& \sigma_{\mathrm{em}}(\lambda)=\frac{\beta}{8 \pi n^{2} \tau_{\mathrm{rad}} c} \\
& \times \frac{\exp (-h c /() k T \lambda)}{\int \lambda^{-4} \sigma_{\mathrm{abs}}(\lambda) \exp (-h c /(k T \lambda)) d \lambda} \sigma_{\mathrm{abs}}(\lambda) .
\end{aligned}
$$


Таблица 5. Прогнозируемая вероятность спонтанного перехода $(A)$, общая вероятность спонтанного перехода $\left(\sum A\right)$, коэффициенты ветвления $(\beta)$ и радиационные времена жизни $\left(\tau_{\text {rad }}\right)$ стекла $P 5$ для возбужденных состояний иона $\mathrm{Tm}^{3+}$

\begin{tabular}{|c|c|c|c|c|c|c|c|}
\hline Переход & $\lambda, \mathrm{nm}$ & $S_{\text {ed }}, 10^{-20} \mathrm{~cm}^{2}$ & $S_{\mathrm{md}}, 10^{-20} \mathrm{~cm}^{2}$ & $A_{\mathrm{rad}}, s^{-1}$ & $\sum A_{\mathrm{rad}}, \mathrm{s}^{-1}$ & $\beta, \%$ & $\tau_{\mathrm{rad}}, \mathrm{ms}$ \\
\hline${ }^{3} F_{4} \rightarrow{ }^{3} \mathrm{H}_{6}$ & 1711 & 2.7310 & 0 & 117.03 & 117.03 & 100.0 & 8.54 \\
\hline $\begin{array}{l}{ }^{3} H_{5} \rightarrow{ }^{3} F_{4} \\
{ }^{3} H_{5} \rightarrow{ }^{3} H_{6}\end{array}$ & $\begin{array}{l}3942 \\
1193\end{array}$ & $\begin{array}{l}1.4669 \\
1.3779\end{array}$ & $\begin{array}{c}0 \\
0.4021\end{array}$ & $\begin{array}{c}4.20 \\
189.73\end{array}$ & 193.93 & $\begin{array}{r}2.16 \\
97.84\end{array}$ & 5.16 \\
\hline $\begin{array}{l}{ }^{3} H_{4} \rightarrow{ }^{3} H_{5} \\
{ }^{3} H_{4} \rightarrow{ }^{3} F_{4} \\
{ }^{3} H_{4} \rightarrow{ }^{3} H_{6}\end{array}$ & $\begin{array}{r}2304 \\
1454 \\
786\end{array}$ & $\begin{array}{l}0.8968 \\
0.7499 \\
1.3866\end{array}$ & $\begin{array}{c}0.2348 \\
0 \\
0\end{array}$ & $\begin{array}{r}20.35 \\
52.41 \\
617.76\end{array}$ & 690.52 & $\begin{array}{r}2.95 \\
7.59 \\
89.46\end{array}$ & 1.45 \\
\hline $\begin{array}{l}{ }^{3} F_{3} \rightarrow{ }^{3} H_{4} \\
{ }^{3} F_{3} \rightarrow{ }^{3} H_{5} \\
{ }^{3} F_{3} \rightarrow{ }^{3} F_{4} \\
{ }^{3} F_{3} \rightarrow{ }^{3} H_{6}\end{array}$ & $\begin{array}{r}5272 \\
1603 \\
1140 \\
684\end{array}$ & $\begin{array}{l}1.1162 \\
1.9715 \\
0.2002 \\
1.5323\end{array}$ & $\begin{array}{c}0 \\
0 \\
0.1556 \\
0\end{array}$ & $\begin{array}{c}2.10 \\
132.14 \\
70.07 \\
1335.86\end{array}$ & 1540.16 & $\begin{array}{r}0.14 \\
8.58 \\
4.55 \\
86.73\end{array}$ & 0.65 \\
\hline $\begin{array}{l}{ }^{3} F_{2} \rightarrow{ }^{3} F_{3} \\
{ }^{3} F_{2} \rightarrow{ }^{3} H_{4} \\
{ }^{3} F_{2} \rightarrow{ }^{3} H_{5} \\
{ }^{3} F_{2} \rightarrow{ }^{3} F_{4} \\
{ }^{3} F_{2} \rightarrow{ }^{3} H_{6}\end{array}$ & $\begin{array}{r}18030 \\
4079 \\
1472 \\
1072 \\
659\end{array}$ & $\begin{array}{l}0.1402 \\
1.0188 \\
1.1928 \\
0.8013 \\
0.2981\end{array}$ & $\begin{array}{l}0 \\
0 \\
0 \\
0 \\
0\end{array}$ & $\begin{array}{c}0.01 \\
5.79 \\
144.61 \\
252.17 \\
407.19\end{array}$ & 809.76 & $\begin{array}{r}0.00 \\
0.72 \\
17.86 \\
31.14 \\
50.28\end{array}$ & 1.23 \\
\hline $\begin{array}{l}{ }^{1} G_{4} \rightarrow{ }^{3} F_{2} \\
{ }^{1} G_{4} \rightarrow{ }^{3} F_{3} \\
{ }^{1} G_{4} \rightarrow{ }^{3} H_{4} \\
{ }^{1} G_{4} \rightarrow{ }^{3} H_{5} \\
{ }^{1} G_{4} \rightarrow{ }^{3} F_{4} \\
{ }^{1} G_{4} \rightarrow{ }^{3} H^{6}\end{array}$ & $\begin{array}{r}1615 \\
1482 \\
1157 \\
770 \\
644 \\
468\end{array}$ & $\begin{array}{l}0.2032 \\
0.5174 \\
0.7966 \\
0.8043 \\
0.1240 \\
0.2524\end{array}$ & $\begin{array}{l}0 \\
0 \\
0 \\
0 \\
0 \\
0\end{array}$ & $\begin{array}{c}10.36 \\
34.15 \\
110.69 \\
381.29 \\
100.92 \\
540.65\end{array}$ & 1178.05 & $\begin{array}{r}0.88 \\
2.90 \\
9.40 \\
32.37 \\
8.57 \\
45.89\end{array}$ & 0.85 \\
\hline $\begin{aligned}{ }^{1} D_{2} & \rightarrow{ }^{1} G_{4} \\
{ }^{1} D_{2} & \rightarrow{ }^{3} F_{2} \\
{ }^{1} D_{2} & \rightarrow{ }^{3} F_{3} \\
{ }^{1} D_{2} & \rightarrow{ }^{3} H_{4} \\
{ }^{1} D_{2} & \rightarrow{ }^{3} H_{5} \\
{ }^{1} D_{2} & \rightarrow{ }^{3} F_{4} \\
{ }^{1} D_{2} & \rightarrow{ }^{3} H_{6}\end{aligned}$ & $\begin{array}{r}1542 \\
789 \\
756 \\
661 \\
514 \\
454 \\
359\end{array}$ & $\begin{array}{l}0.7378 \\
0.6849 \\
0.4562 \\
0.5293 \\
0.0235 \\
1.4001 \\
0.6737\end{array}$ & $\begin{array}{l}0 \\
0 \\
0 \\
0 \\
0 \\
0 \\
0\end{array}$ & $\begin{array}{c}77.79 \\
542.98 \\
411.48 \\
716.42 \\
68.05 \\
5921.18 \\
5818.87\end{array}$ & 13556.77 & $\begin{array}{r}0.57 \\
4.01 \\
3.04 \\
5.28 \\
0.50 \\
43.68 \\
42.92\end{array}$ & 0.07 \\
\hline
\end{tabular}

Таблица 6. Характеристики перехода ${ }^{3} F_{4} \rightarrow{ }^{3} H_{6}$ иона $\mathrm{Tm}^{3+}$ для стекол с различным содержанием $\mathrm{Ba}\left(\mathrm{PO}_{3}\right)_{2}$

\begin{tabular}{c|c|c|c}
\hline $\mathrm{Ba}\left(\mathrm{PO}_{3}\right)_{2}, \mathrm{~mol} . \%$ & $S_{\text {ed }}, 10^{-20} \mathrm{~cm}^{2}$ & $A_{\text {rad }}, \mathrm{s}^{-1}$ & $\tau_{\text {rad }}, \mathrm{ms}$ \\
\hline 2 & 2.4732 & 100.47 & 9.95 \\
5 & 2.7310 & 117.03 & 8.54 \\
10 & 2.4625 & 108.59 & 9.21 \\
20 & 3.6131 & 173.44 & 5.77 \\
30 & 4.2222 & 201.17 & 4.97
\end{tabular}

Полученные сечения излучения для перехода ${ }^{3} F_{4} \rightarrow{ }^{3} H_{6}$ ионов тулия в стеклах, содержащих разные концентрации фосфата, представлены на рис. 3. Было показано, что увеличение содержания фосфатов в стекле увеличивает сечение вынужденного излучения.

Характеристики коэффициента усиления $G(\lambda)$ непосредственно связаны с сечениями поглощения $\sigma_{\mathrm{abs}}$ и излучения $\sigma_{\mathrm{em}} . G(\lambda)$ является функцией инверсии насе- ленности, как видно из соотношения

$$
G(\lambda)=N\left[\gamma \sigma_{\mathrm{em}}-(1-\gamma) \sigma_{\mathrm{abs}}\right],
$$

где $\gamma$ - нормированная населенность верхнего лазерного уровня, определяемая интенсивностью накачки.

На рис. 4, $а$ показан коэффициент усиления для перехода ${ }^{3} F_{4} \rightarrow 3 H_{6}$ в стекле $P 5$. Сравнение формы полосы усиления стекла $P 5$ с полученным в [4] коэффициентом усиления силикатного стекла, активированного ионами тулия (рис. $4, b)$ показало, что фторофосфатное имеет более широкую бесструктурную полосу усиления.

\section{Выводы}

В рамках теории Джадда-Офельта проведен анализ спектрально-люминесцентных параметров фторофосфатных стекол, активированных ионами тулия, при изменении содержания фосфатов. Показаны близость значе- 

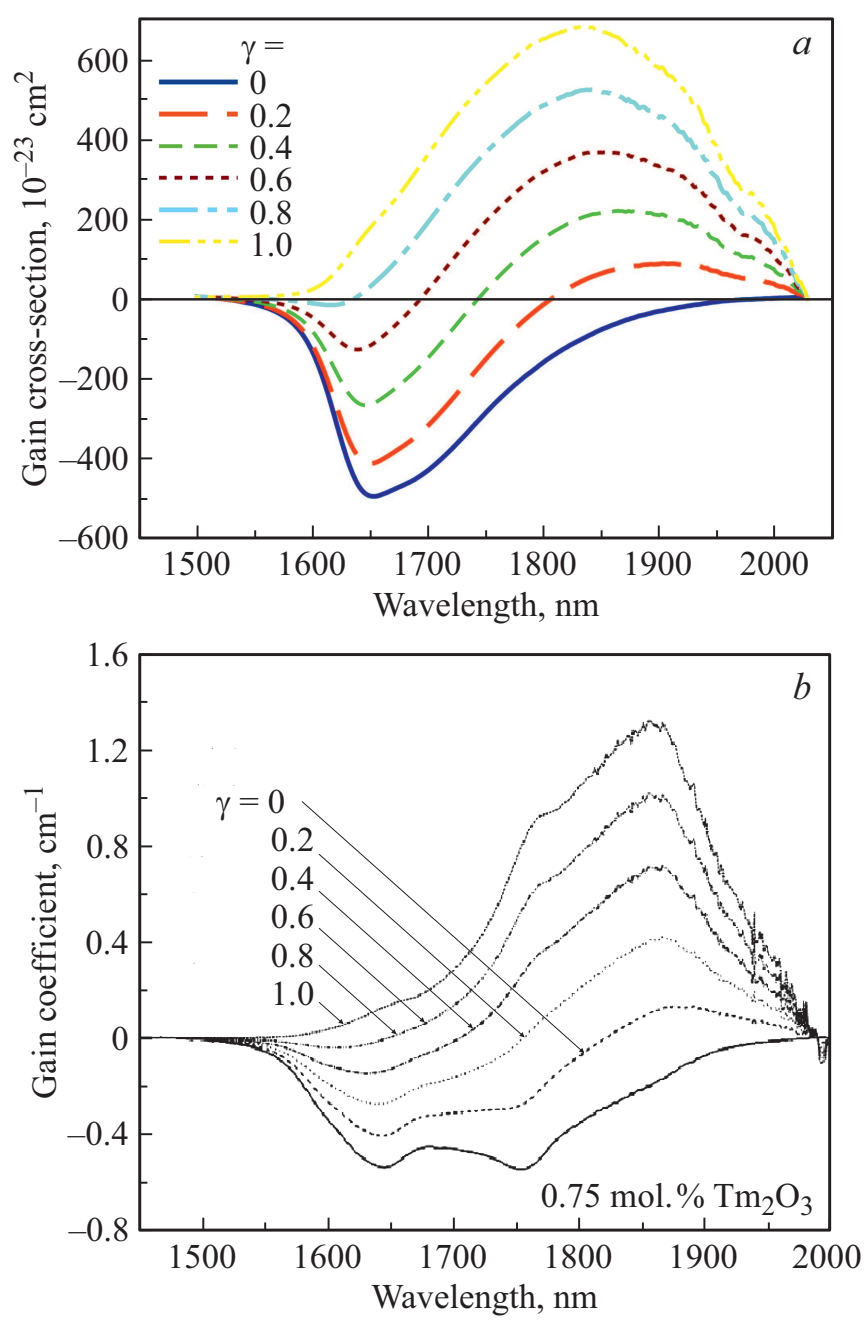

Рис. 4. Сечение усиления стекла $P 5(a)$ и коэффициент усиления силикатного стекла, легированного $\mathrm{Tm}^{3+}(b)$ [4], при различных значениях $\gamma-$ нормализованной заселенности верхнего лазерного уровня, определяемой интенсивностью накачки.

ний параметров $\Omega_{t}$ стекол с содержанием до $10 \mathrm{~mol} \%$ $\mathrm{Ba}\left(\mathrm{PO}_{3}\right)_{2}$ к аналогичным параметрам фторидных стекол и резкое увеличение их значений при дальнейшем увеличения фосфатов. Обнаружено, что при малых концентрациях фосфатов для стекол характерно высокое радиационное время жизни возбужденного лазерного уровня ${ }^{3} F_{4}$. Для исследованных стекол характерен широкий бесструктурный спектр коэффициента усиления, что делает их перспективными материалами для создания широкополосных усилителей, работающих в диапазоне $1800-2000 \mathrm{~nm}$.

\section{Финансирование работы}

Работа проводилась при финансовой поддержке РНФ (соглашение № 19-13-00343).

\section{Конфликт интересов}

Авторы заявляют, что у них нет конфликта интересов.

\section{Список литературы}

[1] Jackson S.D., Sabella A., Hemming A., Bennetts S., Lancaster D.G. // Opt. Lett. 2007. V. 32. N 3. P. 241-243. doi 10.1364/OL.32.000241

[2] Jackson S.D. // Laser Photon. Rev. 2009. V. 3. N 5. P. 466-482. doi 10.1002/lpor.200810058

[3] Richards B., Tsang Y., Binks D., Lousteau J., Jha A. // Opt. Lett. 2008. V. 33. N 4. P. 402-404. doi 10.1364/OL.33.000402

[4] Li M., Bai G., Guo Y., Hu L., Zhang J. // J. Lumin. 2012. V. 132. N 7. P. $1830-1835$. doi 10.1016/J.JLUMIN.2012.02.022

[5] Peng B., Izumitani T. // Opt. Mater. 1995. V. 4. P. 797-810.

[6] Li K., Zhang Q., Bai G., Fan S., Zhang J., Hu L. // J. Alloys Compd. 2010. V. 504. N 2. P. 573-578. doi 10.1016/J.JALLCOM.2010.05.162.

[7] Xu R.R., Tian Y., Wang M., Hu L.L., Zhang J.J. // Appl. Phys. B. 2011. V. 102. N 1. P. 109-116. doi $10.1007 / \mathrm{s} 00340-$ 010-4115-6

[8] Kermaoui A., Pellé F. // J. Alloys Compd. 2009. V. 469. N 1-2. P. 601-608. doi 10.1016/J.JALLCOM.2008.02.024

[9] Nazabal V., Poulain M., Olivier M., Pirasteh P., Camy P., Doualan J.-L., Guy S., Djouama T., Boutarfaia A., Adam J.L. // J. Fluor. Chem. 2012. V. 134. P. 18-23. doi 10.1016/j.jfluchem.2011.06.035

[10] Ehrt D. // Curr. Opin. Solid State Mater. Sci. 2003. V. 7. N 2. P. 135-141. doi 10.1016/S1359-0286(03)00049-4

[11] Kolobkova E., Alkhlef A., Dinh B.M., Yasukevich A.S., Dernovich O.P., Kuleshov N.V., Nikonorov N. // J. Lumin. 2019. V. 206. P. 523-529. doi 10.1016/j.jlumin.2018.10.082

[12] Kolobkova E., Alkhlef A., Nikonorov N. //Proc. SPIE. 2020. V. 11357, Fiber Lasers and Glass Photonics: Materials through Applications II, 113571V (1 April 2020). doi $10.1117 / 12.2555856$

[13] Kolobkova E., Alkhlef A., Mironov L.Yu., Bogdanov O. // Ceramics International. 2020. doi 10.1016/j.ceramint.2020.04.221

[14] Kolobkova E., Alkhlef A., Yasukevich A., Babkina A. // Opt. Mat. Express. 2019. V. 9. P. 3666-3679. doi 10.1364/OME.9.003666D

[15] Binnemans K., Van Deun R., Görller-Walrand C., Adam J.L. // J. Non. Cryst. Solids. 1998. V. 238. N 1. P. 11-29. doi 10.1016/S0022-3093(98)00540-7

[16] Zhang G., Poulain M.J.// J. Alloys Compd. 1998. P. 275-277. P. 15-20. doi 10.1016/S0925-8388(98)00265-5

[17] Kolobkova E., Kuleshov N.V., Nikonorov N., Yasukevich A.S., Babkina A. // J. Non-Cryst. Solids. 2019. V. 526. P. 119703. doi 10.1016/j.jnoncrysol.2019.119703

[18] Walsh B.M., Barnes N.P., Reichle D.J., Jiang S. // J. Non. Cryst. Solids. 2006. V. 352. P. 5344-5352. doi 10.1016/j.jnoncrysol.2006.08.029

[19] Carnall W.T., Fields P.R., Rajnak K. // J. Chem. Phys. 1968. V. 49. N 10. P. 4424-4442. doi 10.1063/1.1669893

[20] Wachtler M., Speghini A., Gatterer K., Fritzer H.P., Ajò D., Bettinelli M. // J. Am. Ceram. Soc. 1998. V. 81. N 8. P. 2045-2052. doi 10.1111/j.1151-2916.1998.tb02586.x 
[21] Ebendorff-Heidepriem H., Ehrt D., Bettinelli M., Speghini A. // J. Non. Cryst. Solids. 1998. V. 240. N 1-3. P. 66-78. doi 10.1016/S0022-3093(98)00706-6

[22] Liao M.S., Fang Y.Z., Sun H.T., Hu L.L. // Opt. Mater. 2007. V. 29. N 7. P. 867-872. doi 10.1016/J.OPTMAT.2006.01.012

[23] Tian Y., Xu R., Zhang L., Hu L., Zhang J. // J. Appl. Phys. 2010. V. 108. N 8. P. 83504. doi 10.1063/1.3499283

[24] Zhang Q., Chen G., Zhang G., Qiu J., Chen D. // J. Appl. Phys. 2009. V. 106. N 11. P. 113102. doi 10.1063/1.3264882

[25] Yasyukevich A.S., Shcherbitskii V.G., Kisel' V.É., Mandrik A.V., Kuleshov N.V. // J. Appl. Spectrosc. 2004. V. 71. N 2. P. $202-208$. doi 10.1023/B:JAPS.0000032875.04400.a0 